\title{
Extremal values on Zagreb indices of trees with given distance $k$-domination number
}

\author{
Lidan Pei and Xiangfeng Pan*
}

"Correspondence: xfpan@ustc.edu School of Mathematical Sciences, Anhui University, Hefei, Anhui, 230601, China

\section{Springer}

\begin{abstract}
Let $G=(V(G), E(G))$ be a graph. A set $D \subseteq V(G)$ is a distance $k$-dominating set of $G$ if for every vertex $u \in V(G) \backslash D, d_{G}(u, v) \leq k$ for some vertex $v \in D$, where $k$ is a positive integer. The distance $k$-domination number $\gamma_{k}(G)$ of $G$ is the minimum cardinality among all distance $k$-dominating sets of $G$. The first Zagreb index of $G$ is defined as $M_{1}=\sum_{u \in V(G)} d^{2}(u)$ and the second Zagreb index of $G$ is $M_{2}=\sum_{u v \in E(G)} d(u) d(v)$. In this paper, we obtain the upper bounds for the Zagreb indices of $n$-vertex trees with given distance $k$-domination number and characterize the extremal trees, which generalize the results of Borovićanin and Furtula (Appl. Math. Comput. 276:208-218, 2016). What is worth mentioning, for an $n$-vertex tree $T$, is that a sharp upper bound on the distance $k$-domination number $\gamma_{k}(T)$ is determined.
\end{abstract}

MSC: $05 C 35 ; 05 C 69$

Keywords: first Zagreb index; second Zagreb index; trees; distance $k$-domination number

\section{Introduction}

Throughout this paper, all graphs considered are simple, undirected and connected. Let $G=(V, E)$ be a simple and connected graph, where $V=V(G)$ is the vertex set and $E=E(G)$ is the edge set of $G$. The eccentricity of $v$ is defined as $\varepsilon_{G}(v)=\max \left\{d_{G}(u, v) \mid u \in V(G)\right\}$. The diameter of $G$ is $\operatorname{diam}(G)=\max \left\{\varepsilon_{G}(v) \mid v \in V(G)\right\}$. A path $P$ is called a diameter path of $G$ if the length of $P$ is $\operatorname{diam}(G)$. Denote by $N_{G}^{i}(v)$ the set of vertices with distance $i$ from $v$ in $G$, that is, $N_{G}^{i}(v)=\{u \in V(G) \mid d(u, v)=i\}$. In particular, $N_{G}^{0}(v)=\{v\}$ and $N_{G}^{1}(v)=N_{G}(v)$. A vertex $v \in V(G)$ is called a private $k$-neighbor of $u$ with respect to $D$ if $\bigcup_{i=0}^{k} N_{G}^{i}(v) \cap D=$ $\{u\}$. That is, $d_{G}(v, u) \leq k$ and $d_{G}(v, x) \geq k+1$ for any vertex $x \in D \backslash\{u\}$. The pendent vertex is the vertex of degree 1 .

A chemical molecule can be viewed as a graph. In a molecular graph, the vertices represent the atoms of the molecule and the edges are chemical bonds. A topological index of a molecular graph is a mathematical parameter which is used for studying various properties of this molecule. The distance-based topological indices, such as the Wiener index [2, 3] and the Balaban index [4], have been extensively researched for many decades. Meanwhile the spectrum-based indices developed rapidly, such as the Estrada index [5], the Kirchhoff index [6] and matching energy [7]. The eccentricity-based topological indices, such as the eccentric distance sum [8], the connective eccentricity index [9] and the adjacent eccentric distance sum [10], were proposed and studied recently. The degree-based topological

(c) The Author(s) 2018. This article is distributed under the terms of the Creative Commons Attribution 4.0 International License (http://creativecommons.org/licenses/by/4.0/), which permits unrestricted use, distribution, and reproduction in any medium, provided you give appropriate credit to the original author(s) and the source, provide a link to the Creative Commons license, and indicate if changes were made. 
indices, such as the Randić index [11-13], the general sum-connectivity index [14, 15], the Zagreb indices [16], the multiplicative Zagreb indices [17, 18] and the augmented Zagreb index [19], where the Zagreb indices include the first Zagreb index $M_{1}=\sum_{u \in V(G)} d^{2}(u)$ and the second Zagreb index $M_{2}=\sum_{u v \in E(G)} d(u) d(v)$, represent one kind of the most famous topological indices. In this paper, we continue the work on Zagreb indices. Further study about the Zagreb indices can be found in [20-25]. Many researchers are interested in establishing the bounds for the Zagreb indices of graphs and characterizing the extremal graphs $[1,26-40]$.

A set $D \subseteq V(G)$ is a dominating set of $G$ if, for any vertex $u \in V(G) \backslash D, N_{G}(u) \cap D \neq \emptyset$. The domination number $\gamma(G)$ of $G$ is the minimum cardinality of dominating sets of $G$. For $k \in N^{+}$, a set $D \subseteq V(G)$ is a distance $k$-dominating set of $G$ if, for every vertex $u \in V(G) \backslash D$, $d_{G}(u, v) \leq k$ for some vertex $v \in D$. The distance $k$-domination number $\gamma_{k}(G)$ of $G$ is the minimum cardinality among all distance $k$-dominating sets of $G[41,42]$. Every vertex in a minimum distance $k$-dominating set has a private $k$-neighbor. The domination number is the special case of the distance $k$-domination number for $k=1$. Two famous books $[43,44]$ written by Haynes et al. show us a comprehensive study of domination. The topological indices of graphs with given domination number or domination variations have attracted much attention of researchers [1, 45-47].

Borovićanin [1] showed the sharp upper bounds on the Zagreb indices of $n$-vertex trees with domination number $\gamma$ and characterized the extremal trees. Motivated by [1], we describe the upper bounds for the Zagreb indices of $n$-vertex trees with given distance $k$-domination number and find the extremal trees. Furthermore, a sharp upper bound, in terms of $n, k$ and $\Delta$, on the distance $k$-domination number $\gamma_{k}(T)$ for an $n$-vertex tree $T$ is obtained in this paper.

\section{Lemmas}

In this section, we give some lemmas which are helpful to our results.

Lemma $2.1([24,48])$ If $T$ is an n-vertex tree, different from the star $S_{n}$, then $M_{i}(T)<$ $M_{i}\left(S_{n}\right)$ for $i=1,2$.

In what follows, we present two graph transformations that increase the Zagreb indices.

Transformation I ([49]) Let $T$ be an $n$-vertex tree $(n>3)$ and $e=u v \in E(T)$ be a nonpendent edge. Assume that $T-u v=T_{1} \cup T_{2}$ with vertex $u \in V\left(T_{1}\right)$ and $v \in V\left(T_{2}\right)$. Let $T^{\prime}$ be the tree obtained by identifying the vertex $u$ of $T_{1}$ with vertex $v$ of $T_{2}$ and attaching a pendent vertex $w$ to the $u(=v)$ (see Figure 1). For the sake of convenience, we denote $T^{\prime}=\tau(T, u v)$.

Lemma 2.2 Let $T$ be a tree of order $n(\geq 3)$ and $T^{\prime}=\tau(T, u v)$. Then $M_{i}\left(T^{\prime}\right)>M_{i}(T)$, $i=1,2$.

Figure $1 T$ and $T^{\prime}$ in Transformation I.

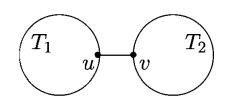

$T$

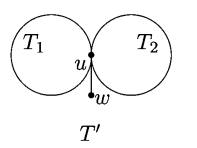


Proof It is obvious that $d_{T^{\prime}}(u)=d_{T}(u)+d_{T}(v)-1$ and

$$
\begin{aligned}
M_{1}\left(T^{\prime}\right)-M_{1}(T) & =\left(d_{T}(u)+d_{T}(v)-1\right)^{2}+1-d_{T}^{2}(u)-d_{T}^{2}(v) \\
& =2\left(d_{T}(u)-1\right)\left(d_{T}(v)-1\right) \\
& >0 .
\end{aligned}
$$

Let $x \in V(T)$ be a vertex different from $u$ and $v$. Then

$$
\begin{aligned}
M_{2}\left(T^{\prime}\right)-M_{2}(T)= & \left(d_{T}(u)+d_{T}(v)-1\right)\left(\sum_{x u \in E\left(T_{1}\right)} d_{T}(x)+\sum_{x v \in E\left(T_{2}\right)} d_{T}(x)+1\right) \\
& -d_{T}(u) \sum_{x u \in E\left(T_{1}\right)} d_{T}(x)-d_{T}(v) \sum_{x v \in E\left(T_{2}\right)} d_{T}(x)-d_{T}(u) d_{T}(v) \\
= & \left(d_{T}(v)-1\right) \sum_{x u \in E\left(T_{1}\right)} d_{T}(x)+\left(d_{T}(u)-1\right) \sum_{x v \in E\left(T_{2}\right)} d_{T}(x) \\
& +d_{T}(u)+d_{T}(v)-1-d_{T}(u) d_{T}(v) \\
\geq & 2\left(d_{T}(v)-1\right)\left(d_{T}(u)-1\right)+d_{T}(u)+d_{T}(v)-1-d_{T}(u) d_{T}(v) \\
= & \left(d_{T}(v)-1\right)\left(d_{T}(u)-1\right) \\
> & 0 .
\end{aligned}
$$

This completes the proof.

Lemma 2.3 ([50]) Let $u$ and $v$ be two distinct vertices in $G . u_{1}, u_{2}, \ldots, u_{r}$ are the pendent vertices adjacent to $u$ and $v_{1}, v_{2}, \ldots, v_{t}$ are the pendent vertices adjacent to $v$. Define $G^{\prime}=G-$ $\left\{v v_{1}, v v_{2}, \ldots, v v_{t}\right\}+\left\{u v_{1}, u v_{2}, \ldots, u v_{t}\right\}$ and $G^{\prime \prime}=G-\left\{u u_{1}, u u_{2}, \ldots, u u_{r}\right\}+\left\{v u_{1}, v u_{2}, \ldots, v u_{r}\right\}$, as shown in Figure 2. Then either $M_{i}\left(G^{\prime}\right)>M_{i}(G)$ or $M_{i}\left(G^{\prime \prime}\right)>M_{i}(G), i=1,2$.

Lemma 2.4 ([51]) For a connected graph G of order $n$ with $n \geq k+1, \gamma_{k}(G) \leq\left\lfloor\frac{n}{k+1}\right\rfloor$.

Let $G$ be a connected graph of order $n$. If $\gamma_{k}(G) \geq 2$, then $n \geq k+1$. Otherwise, $\gamma_{k}(G)=1$, a contradiction. Hence, by Lemma 2.4, we have $\gamma_{k}(G) \leq\left\lfloor\frac{n}{k+1}\right\rfloor$ and $n \geq(k+1) \gamma_{k}$ for any connected graph $G$ of order $n$ if $\gamma_{k}(G) \geq 2$.

Lemma 2.5 Let $T$ be an $n$-vertex tree with distance $k$-domination number $\gamma_{k} \geq 2$. Then $\Delta \leq n-k \gamma_{k}$.

Proof Suppose that $\Delta \geq n-k \gamma_{k}+1$. Let $v \in V(T)$ be the vertex such that $d(v)=\Delta$ and $N(v)=\left\{v_{1}, \ldots, v_{\Delta}\right\}$. Denote by $T^{i}$ the component of $T-v$ containing the vertex $v_{i}, i=$

Figure $2 G, G^{\prime}$ and $G^{\prime \prime}$ in Lemma 2.3.

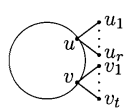

$G$

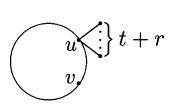

$G^{\prime}$

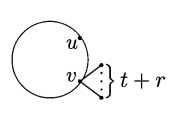

$G^{\prime \prime}$ 
$1, \ldots, \triangle$. Let $D$ be a minimum distance $k$-dominating set of $T$,

$$
S_{1}=\left\{i \mid i \in\{1,2, \ldots, \triangle\}, 0 \leq \varepsilon_{T^{i}}\left(v_{i}\right) \leq k-1\right\}
$$

and

$$
S_{2}=\left\{i \mid i \in\{1,2, \ldots, \triangle\}, \varepsilon_{T^{i}}\left(v_{i}\right) \geq k\right\}
$$

Clearly, $\left|S_{2}\right| \geq 1$. If not, $\{v\}$ is a distance $k$-dominating set of $T$, which contradicts $\gamma_{k} \geq 2$. If $\left|S_{1}\right|=0$, then $\varepsilon_{T^{i}}\left(v_{i}\right) \geq k$ for $i=1, \ldots, \Delta$, so $\left|V\left(T^{i}\right) \cap D\right| \geq 1$. Therefore, $\gamma_{k} \geq \Delta \geq$ $n-k \gamma_{k}+1$, which implies that $\gamma_{k} \geq \frac{n+1}{k+1}$. Since $\gamma_{k} \geq 2, \gamma_{k} \leq\left\lfloor\frac{n}{k+1}\right\rfloor$ by Lemma 2.4, a contradiction. Thus, $\left|S_{1}\right| \geq 1$. Let $i_{1} \in S_{1}$ and

$$
\varepsilon_{T^{i_{1}}}\left(v_{i_{1}}\right)=\max \left\{\varepsilon_{T^{i}}\left(v_{i}\right) \mid i \in S_{1}\right\}=\lambda .
$$

Then $0 \leq \lambda \leq k-1$, so $\left|S_{2}\right| \leq\left\lfloor\frac{n-\Delta-1-\lambda}{k}\right\rfloor \leq\left\lfloor\frac{k \gamma_{k}-2}{k}\right\rfloor \leq \gamma_{k}-1$.

If $V\left(T^{i}\right) \cap D=D_{1} \neq \emptyset$ for some $i \in S_{1}$, then $D-D_{1}+\{v\}$ is a distance $k$-dominating set according to the definition of $S_{1}$. Thus, we assume that $V\left(T^{i}\right) \cap D=\emptyset$ for each $i \in S_{1}$. Similarly, suppose that $D^{\prime} \cap V\left(T^{i_{1}}\right)=\emptyset$ where $D^{\prime}$ is a minimum distance $k$-dominating set of the tree $T^{\prime}=T-\bigcup_{i \in S_{1} \backslash\left\{i_{1}\right\}} V\left(T^{i}\right)$.

We claim that $D^{\prime}$ is a distance $k$-dominating set of $T$. Let $y \in V\left(T^{i_{1}}\right)$ be the vertex such that $d\left(v_{i_{1}}, y\right)=\lambda$ and $y^{\prime} \in D_{1}^{\prime}=\bigcup_{i=0}^{k} N_{T^{\prime}}^{i}(y) \cap D^{\prime}$. Then $y^{\prime} \in V\left(T^{\prime}\right) \backslash V\left(T^{i_{1}}\right)$ and $d\left(y, y^{\prime}\right)=d(y, v)+d\left(v, y^{\prime}\right) \leq k$, so, for $x \in \bigcup_{i \in S_{1} \backslash\left\{i_{1}\right\}} V\left(T^{i}\right)$, we have $d\left(x, y^{\prime}\right)=d(x, v)+d\left(v, y^{\prime}\right) \leq$ $d(y, v)+d\left(v, y^{\prime}\right) \leq k$. Hence, all the vertices in $\bigcup_{i \in S_{1} \backslash\left\{i_{1}\right\}} V\left(T^{i}\right)$ can be dominated by $y^{\prime} \in D^{\prime}$. Therefore, $D^{\prime}$ is a distance $k$-dominating set of $T$, so the claim is true.

In view of

$$
k+1<(k+1)\left|S_{2}\right|+\lambda+2 \leq\left|V\left(T^{\prime}\right)\right| \leq n-\left|S_{1}\right|+1=n-\Delta+\left|S_{2}\right|+1,
$$

one has

$$
\begin{aligned}
\gamma_{k} & \leq\left|D^{\prime}\right| \\
& \leq\left\lfloor\frac{n-\Delta+\left|S_{2}\right|+1}{k+1}\right\rfloor \quad(\text { by Lemma 2.4) } \\
& \leq\left\lfloor\frac{(k+1) \gamma_{k}-1}{k+1}\right\rfloor \quad\left(\text { since } \Delta \geq n-k \gamma_{k}+1,\left|S_{2}\right| \leq \gamma_{k}-1\right) \\
& <\gamma_{k},
\end{aligned}
$$

a contradiction as desired.

Determining the bound on the distance $k$-domination number of a connected graph is an attractive problem. In Lemma 2.5, an upper bound for the distance $k$-domination number of a tree is characterized. Namely, if $T$ is an $n$-vertex tree with distance $k$-domination number $\gamma_{k} \geq 2$, then $\gamma_{k}(T) \leq \frac{n-\Delta(T)}{k}$.

Let $\mathcal{T}_{n, k, \gamma_{k}}$ be the set of all $n$-vertex trees with distance $k$-domination number $\gamma_{k}$ and $S_{n-k \gamma_{k}+1}$ be the star of order $n-k \gamma_{k}+1$ with pendent vertices $v_{1}, v_{2}, \ldots, v_{n-k \gamma_{k}}$. Denote by $T_{n, k, \gamma_{k}}$ the tree formed from $S_{n-k \gamma_{k}}$ by attaching a path $P_{k-1}$ to $v_{1}$ and attaching a path 
Figure $3 T_{n, k, y_{k}}$.

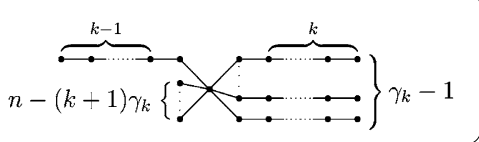

$P_{k}$ to $v_{i}$ for each $i \in\left\{2, \ldots, \gamma_{k}\right\}$, as shown in Figure 3. Then $T_{n, k, \gamma_{k}} \in \mathcal{T}_{n, k, \gamma_{k}}$. Even more noteworthy is the notion that $\gamma_{k}\left(T_{n, k, \gamma_{k}}\right)=\gamma_{k}=\frac{n-\Delta\left(T_{n, k, \gamma_{k}}\right)}{k}$. It implies that the upper bound on the distance $k$-domination number mentioned in the above paragraph is sharp.

The Zagreb indices of $T_{n, k, \gamma_{k}}$ are computed as

$$
M_{1}\left(T_{n, k, \gamma_{k}}\right)=\left(n-k \gamma_{k}\right)\left(n-k \gamma_{k}+1\right)+4\left(k \gamma_{k}-1\right)
$$

and

$$
M_{2}\left(T_{n, k, \gamma_{k}}\right)= \begin{cases}\left(n-k \gamma_{k}\right)\left[n-(k-1) \gamma_{k}\right]+(4 k-2) \gamma_{k}-4 & \text { if } k \geq 2, \\ 2(n-\gamma+1)(\gamma-1)+(n-\gamma)(n-2 \gamma+1) & \text { if } k=1 .\end{cases}
$$

For $k=1$, the distance $k$-domination number $\gamma_{1}(G)$ is the domination number $\gamma(G)$. Furthermore, the upper bounds on the Zagreb indices of an $n$-vertex tree with domination number were studied in [1], so we only consider $k \geq 2$ in the following.

Lemma $2.6([52]) T$ be a tree on $(k+1) n$ vertices. Then $\gamma_{k}(T)=n$ if and only if at least one of the following conditions holds:

(1) $T$ is any tree on $k+1$ vertices;

(2) $T=R \circ k$ for some tree $R$ on $n \geq 1$ vertices, where $R \circ k$ is the graph obtained by taking one copy of $R$ and $|V(R)|$ copies of the path $P_{k-1}$ of length $k-1$ and then joining the ith vertex of $R$ to exactly one end vertex in the ith copy of $P_{k-1}$.

Lemma 2.7 Let $T$ be an $n$-vertex tree with distance $k$-domination number $\gamma_{k}(T) \geq 3$. If $n=(k+1) \gamma_{k}$, then

$$
M_{1}(T) \leq \gamma_{k}\left(\gamma_{k}+1\right)+4\left(k \gamma_{k}-1\right)
$$

and

$$
M_{2}(T) \leq 2 \gamma_{k}^{2}+(4 k-2) \gamma_{k}-4
$$

with equality if and only if $T \cong T_{n, k, \gamma_{k}}$.

Proof When $n=(k+1) \gamma_{k}, T=R \circ k$ for some tree $R$ on $\gamma_{k}$ vertices by Lemma 2.6. Assume that $V(R)=\left\{v_{1}, \ldots, v_{\gamma_{k}}\right\}$. Then $d_{R}\left(v_{i}\right)=d_{T}\left(v_{i}\right)-1$. It is well known that $\sum_{i=1}^{n} d\left(u_{i}\right)=2(n-1)$ for any $n$-vertex tree with vertex set $\left\{u_{1}, \ldots, u_{n}\right\}$. Hence, $\sum_{i=1}^{\gamma_{k}} d_{R}\left(v_{i}\right)=2\left(\gamma_{k}-1\right)$. By the definition of the first Zagreb index, we have

$$
\begin{aligned}
M_{1}(T) & =\sum_{i=1}^{\gamma_{k}} d_{T}^{2}\left(v_{i}\right)+\sum_{x \in V(T) \backslash V(R)} d_{T}^{2}(x) \\
& =\sum_{i=1}^{\gamma_{k}}\left(d_{T}\left(v_{i}\right)-1\right)^{2}+\sum_{x \in V(T) \backslash V(R)} d_{T}^{2}(x)+2 \sum_{i=1}^{\gamma_{k}}\left(d_{T}\left(v_{i}\right)-1\right)+\gamma_{k}
\end{aligned}
$$




$$
\begin{aligned}
& =M_{1}(R)+4(k-1) \gamma_{k}+\gamma_{k}+2 \sum_{i=1}^{\gamma_{k}} d_{R}\left(v_{i}\right)+\gamma_{k} \\
& \leq M_{1}\left(S_{\gamma_{k}}\right)+4(k-1) \gamma_{k}+2 \gamma_{k}+4\left(\gamma_{k}-1\right) \\
& =\gamma_{k}\left(\gamma_{k}+1\right)+4\left(k \gamma_{k}-1\right) .
\end{aligned}
$$

The equality holds if and only if $R \cong S_{\gamma_{k}}$, that is, $T \cong T_{n, k, \gamma_{k}}$. We have

$$
\begin{aligned}
M_{2}(T)= & \sum_{x y \in E(R)} d_{T}(x) d_{T}(y)+\sum_{x y \in E(T) \backslash E(R)} d_{T}(x) d_{T}(y) \\
= & \sum_{x y \in E(R)}\left(d_{T}(x)-1\right)\left(d_{T}(y)-1\right)+\sum_{x y \in E(R)}\left(d_{T}(x)+d_{T}(y)-1\right) \\
& +\sum_{x y \in E(T) \backslash E(R)} d_{T}(x) d_{T}(y) \\
= & M_{2}(R)+\sum_{x \in V(R)} d_{T}(x)\left(d_{T}(x)-1\right)-\left(\gamma_{k}-1\right) \\
& +\sum_{x \in V(R)} 2 d_{T}(x)+4(k-2) \gamma_{k}+2 \gamma_{k} \\
= & M_{2}(R)+\sum_{x \in V(R)}\left(d_{T}(x)-1\right)^{2}+3 \sum_{x \in V(R)}\left(d_{T}(x)-1\right)+4 k \gamma_{k}-5 \gamma_{k}-1 \\
= & M_{2}(R)+M_{1}(R)+6\left(\gamma_{k}-1\right)+4 k \gamma_{k}-5 \gamma_{k}+1 \\
\leq & M_{2}\left(S_{\gamma_{k}}\right)+M_{1}\left(S_{\gamma_{k}}\right)+4 k \gamma_{k}+\gamma_{k}-5 \\
= & 2 \gamma_{k}^{2}+(4 k-2) \gamma_{k}-4 .
\end{aligned}
$$

The equality holds if and only if $R \cong S_{\gamma_{k}}$. As a consequence, $T \cong T_{n, k, \gamma_{k}}$.

Lemma 2.8 Let $G$ be a graph which has a maximum value of the Zagreb indices among all n-vertex connected graphs with distance $k$-domination number and $S_{G}=\{v \in V(G) \mid$ $\left.d_{G}(v)=1, \gamma_{k}(G-v)=\gamma_{k}(G)\right\}$. If $S_{G} \neq \emptyset$, then $\left|N_{G}\left(S_{G}\right)\right|=1$.

Proof Suppose that $\left|N_{G}\left(S_{G}\right)\right| \geq 2$ and $u$ and $v$ are two distinct vertices in $N_{G}\left(S_{G}\right)$. $x_{1}, x_{2}, \ldots, x_{r}$ are the pendent vertices adjacent to $u$ and $y_{1}, y_{2}, \ldots, y_{t}$ are the pendent vertices adjacent to $v$, where $r \geq 1$ and $t \geq 1$. Let $D$ be a minimum distance $k$-dominating set of $G$. If $x_{i} \in D$ for some $i \in\{1, \ldots, r\}$, then $D-x_{i}+u$ is a distance $k$-dominating set of $T$. Hence, we assume that $x_{i} \notin D, i=1, \ldots, r$. Similarly, $y_{i} \notin D$ for $1 \leq i \leq t$. Define $G_{1}=G-\left\{v y_{1}\right\}+\left\{u y_{1}\right\}$ and $G_{2}=G-\left\{u x_{1}\right\}+\left\{v x_{1}\right\}$. Then $\gamma_{k}\left(G_{1}\right)=\gamma_{k}\left(G_{2}\right)=\gamma_{k}(G)$. In addition, we have either $M_{i}\left(G_{1}\right)>M_{i}(G)$ or $M_{i}\left(G_{2}\right)>M_{i}(G), i=1,2$, by a similar proof of Lemma 2.3 and thus omitted here (for reference, see the Appendix). It follows a contradiction, as desired.

\section{Main results}

In this section, we give upper bounds on the Zagreb indices of a tree with given order $n$ and distance $k$-domination number $\gamma_{k}$. If $P=v_{0} v_{1} \cdots v_{d}$ is a diameter path of an $n$-vertex tree $T$, then denote by $T_{i}$ the component of $T-\left\{v_{i-1} v_{i}, v_{i} v_{i+1}\right\}$ containing $v_{i}, i=1,2, \ldots, d-1$. By Lemma 2.1, we obtain Theorem 3.1 directly. 
Theorem 3.1 Let $T$ be an n-vertex tree and $\gamma_{k}(T)=1$. Then $M_{1}(T) \leq n(n-1)$ and $M_{2}(T) \leq(n-1)^{2}$. The equality holds if and only if $T \cong S_{n}$.

Let $T_{n, k, 2}^{i}$ be the tree obtained from the path $P_{2 k+2}=v_{0} \cdots v_{2 k+1}$ by joining $n-2(k+1)$ pendent vertices to $v_{i}$, where $i \in\{1, \ldots, 2 k\}$.

Theorem 3.2 If $T$ is an n-vertex tree with distance $k$-domination number $\gamma_{k}(T)=2$, then

$$
M_{1}(T) \leq(n-2 k)(n-2 k+1)+4(2 k-1)
$$

with equality if and only if $T \cong T_{n, k, 2}^{i}$, where $i \in\{1, \ldots, k\}$. Also,

$$
M_{2}(T) \leq(n-2 k)(n-2 k+2)+8 k-8
$$

with equality if and only if $T \cong T_{n, k, 2}^{i}$, where $i \in\{2, \ldots, k\}$.

Proof Assume that $T \in \mathcal{T}_{n, k, 2}$ is the tree that maximizes the Zagreb indices and $P=$ $v_{0} v_{1} \cdots v_{d}$ is a diameter path of $T$. If $d \leq 2 k$, then $\left\{v_{\left\lfloor\frac{d}{2}\right\rfloor}\right\}$ is a distance $k$-dominating set of $T$, a contradiction to $\gamma_{k}(T)=2$. If $d \geq 2 k+2$, define $T^{\prime}=\tau\left(T, v_{i} v_{i+1}\right)$, where $i \in\{1, \ldots, d-2\}$. Then $T^{\prime} \in \mathcal{T}_{n, k, 2}$. By Lemma 2.2, we have $M_{i}\left(T^{\prime}\right)>M_{i}(T), i=1,2$, a contradiction. Hence, $d=2 k+1$.

If $T_{i}$ is not a star for some $i \in\{1,2, \ldots, d-1\}$, then there exists an $n$-vertex tree $T^{\prime}$ in $\mathcal{T}_{n, k, 2}$ such that $M_{i}\left(T^{\prime}\right)>M_{i}(T)$ for $i=1,2$ by Lemma 2.2 , a contradiction. Besides, $T \cong T_{n, k, 2}^{i}$ for some $i \in\{1, \ldots, d-1\}$ by Lemma 2.3 .

Since $M_{1}\left(T_{n, k, 2}^{i}\right)=M_{1}\left(T_{n, k, 2}^{j}\right)$ for $1 \leq i \neq j \leq d-1$ and $T_{n, k, 2}^{i} \cong T_{n, k, 2}^{d-i}$ for $k+1 \leq i \leq d-1$, we get $T \cong T_{n, k, 2}^{i}, i \in\{1, \ldots, k\}$. By direct computation, one has $M_{1}(T)=M_{1}\left(T_{n, k, 2}^{i}\right)=$ $(n-2 k)(n-2 k+1)+4(2 k-1), i \in\{1, \ldots, k\}$. In addition, $M_{2}\left(T_{n, k, 2}^{1}\right)=M_{2}\left(T_{n, k, 2}^{d-1}\right)<$ $M_{2}\left(T_{n, k, 2}^{2}\right)=\cdots=M_{2}\left(T_{n, k, 2}^{d-2}\right)$ and $T_{n, k, 2}^{i} \cong T_{n, k, 2}^{d-i}$ for $i \in\{k+1, \ldots, d-2\}$. Hence, $T \cong T_{n, k, 2}^{i}$, where $i \in\{2, \ldots, k\}$. Moreover, $M_{2}(T)=M_{2}\left(T_{n, k, 2}^{i}\right)=(n-2 k)(n-2 k+2)+8 k-8$. This completes the proof.

Lemma 3.3 Let tree $T \in \widetilde{T}_{n, k, 3}$. Then

$$
M_{1}(T) \leq(n-3 k)(n-3 k+1)+4(3 k-1)
$$

and

$$
M_{2}(T) \leq(n-3 k)(n-3 k+3)+12 k-10,
$$

with equality if and only if $T \cong T_{n, k, 3}$.

Proof Assume that $T \in \mathcal{T}_{n, k, 3}$. We complete the proof by induction on $n$. By Lemma 2.4, we have $n \geq(k+1) \gamma_{k}$. This lemma is true for $n=(k+1) \gamma_{k}$ by Lemma 2.7. Suppose that $n>3(k+1)$ and the statement holds for $n-1$ in the following.

Let $D$ be a minimum distance $k$-dominating set of $T$ and $P=v_{0} v_{1} \cdots v_{d}$ be a diameter path of $T$. Then $d \geq 2 k+2$. Otherwise, $\left\{v_{k}, v_{k+1}\right\}$ is a distance $k$-dominating set, a contradiction. Note that $\bigcup_{i=0}^{k} N_{T}^{i}\left(v_{0}\right) \cap D \neq \emptyset$ and $\bigcup_{i=0}^{k} N_{T}^{i}\left(v_{0}\right) \subseteq\left(\bigcup_{i=0}^{k-1} V\left(T_{i}\right) \cup\left\{v_{k}\right\}\right)$. Hence, 
$\left(\bigcup_{i=0}^{k-1} V\left(T_{i}\right) \cup\left\{v_{k}\right\}\right) \cap D \neq \emptyset$. However, $\bigcup_{i=0}^{k} N_{T}^{i}(x) \subseteq \bigcup_{i=0}^{k} N_{T}^{i}\left(v_{k}\right)$ for $x \in \bigcup_{i=0}^{k} V\left(T_{i}\right) \backslash\left\{v_{k}\right\}$, so we assume that $v_{k} \in D$ and $\left(\bigcup_{i=0}^{k} V\left(T_{i}\right) \backslash\left\{v_{k}\right\}\right) \cap D=\emptyset$. Similarly, $v_{d-k} \in D$ and $\left(\bigcup_{i=d-k}^{d} V\left(T_{i}\right) \backslash\left\{v_{d-k}\right\}\right) \cap D=\emptyset$. Suppose that $v_{0}=u_{1}, v_{d}=u_{2}, \ldots, u_{m}$ are the pendent vertices of $T$ and $S_{T}=\left\{u_{i} \mid 1 \leq i \leq m, \gamma_{k}\left(T-u_{i}\right)=\gamma_{k}(T)\right\}$. We have the following claim.

Claim $1 S_{T} \neq \emptyset$.

Proof Assume that $S_{T}=\emptyset$. Namely, $\gamma_{k}\left(T-u_{i}\right)=\gamma_{k}(T)-1$ for each $i \in\{1, \ldots, m\}$. If $D \backslash\left\{w_{i}\right\}$ is a minimum distance $k$-dominating set of the tree $T-u_{i}$, where $w_{i} \in D$, then $w_{i} \neq w_{j}$ for $1 \leq i \neq j \leq m$. Otherwise, $\gamma_{k}\left(T-u_{i}\right)=\gamma_{k}(T)$ or $\gamma_{k}\left(T-u_{j}\right)=\gamma_{k}(T)$, a contradiction. It follows that $m \leq \gamma_{k}$.

If $d_{T}\left(v_{i}\right) \geq 3$ for some $i \in\{2, \ldots, k, d-k, \ldots, d-1\}$, then $V\left(T_{i}\right) \cap\left\{u_{3}, \ldots, u_{m}\right\} \neq \emptyset$. In view of $\left\{v_{k}, v_{d-k}\right\} \subseteq D$, we have $\gamma_{k}(T-x)=\gamma_{k}(T)$ for $x \in V\left(T_{i}\right) \cap\left\{u_{3}, \ldots, u_{m}\right\}$, a contradiction. Hence, $d_{T}\left(v_{i}\right)=2$ for $i \in\{2, \ldots, k, d-k, \ldots, d-1\}$.

Since $\gamma_{k}\left(T-v_{0}\right)=\gamma_{k}(T)-1, v_{1}$ must be dominated by the vertices in $D \backslash\left\{v_{k}\right\}$. Bearing in mind that $\left(\bigcup_{i=0}^{k} V\left(T_{i}\right) \backslash\left\{v_{k}\right\}\right) \cap D=\emptyset$, one has $v_{k+1} \in D$. The same applies to $v_{d-k-1}$. Hence, $\left\{v_{k}, v_{k+1}, v_{d-k-1}, v_{d-k}\right\} \subseteq D$. If $d>2 k+2$, then the vertices $v_{k}, v_{k+1}, v_{d-k-1}$ and $v_{d-k}$ are different from each other, a contradiction to $\gamma_{k}(T)=3$. As a consequence, $d=2 k+2$ and thus $D=\left\{v_{k}, v_{k+1}, v_{d-k}\right\}$.

If $d_{T}\left(v_{k+1}\right)=2$, then $T \cong P_{2 k+3}$ and $\left\{v_{k}, v_{d-k}\right\}$ is a distance $k$-dominating set, a contradiction. It follows that $d_{T}\left(v_{k+1}\right) \geq 3$. Hence, $m \geq 3=\gamma_{k}$. Recalling that $m \leq \gamma_{k}=3$, we have $m=3$, which implies that $T_{k+1}$ is a path with end vertices $v_{k+1}$ and $u_{3}$. If $d\left(v_{k+1}, u_{3}\right)>k$, then $u_{3}$ cannot be dominated by the vertices in $D$. If $d\left(v_{k+1}, u_{3}\right)<k$, then $D \backslash\left\{v_{k+1}\right\}$ is a distance $k$-dominating set, a contradiction. Therefore, $d\left(v_{k+1}, u_{3}\right)=k$. We conclude that $|V(T)|=3(k+1)$, which contradicts $n>3(k+1)$, so Claim 1 is true.

Considering $S_{T} \neq \emptyset$ for $T \in \mathcal{T}_{n, k, 3}$, the tree among $\mathcal{T}_{n, k, 3}$ that maximizes the Zagreb indices must be in the set $\left\{T^{*} \in \mathcal{T}_{n, k, 3}|| N_{T^{*}}\left(S_{T^{*}}\right) \mid=1\right\}$ by Lemma 2.8 . To determine the extremal trees among $\mathcal{T}_{n, k, 3}$, we assume that $T \in\left\{T^{*} \in \mathcal{T}_{n, k, 3}|| N_{T^{*}}\left(S_{T^{*}}\right) \mid=1\right\}$ in what follows.

Let $u_{i}$ be a pendent vertex such that $\gamma_{k}\left(T-u_{i}\right)=\gamma_{k}(T)$ and $s$ be the unique vertex adjacent to $u_{i}$. By Lemma 2.5, $d_{T}(s) \leq \Delta \leq n-k \gamma_{k}$. Define $A=\left\{x \in V(T) \mid d_{T}(x)=1, x s \notin E(T)\right\}$ and $B=\left\{x \in V(T) \mid d_{T}(x) \geq 2, x s \notin E(T)\right\}$. Then $\gamma_{k}(T-x)=\gamma_{k}(T)-1$ for all $x \in A$. As a consequence, $|A| \leq \gamma_{k}$ from the proof of Claim 1. By the induction hypothesis,

$$
\begin{aligned}
M_{1}(T) & =M_{1}\left(T-u_{i}\right)+2 d(s) \\
& \leq\left(n-1-k \gamma_{k}\right)\left(n-1-k \gamma_{k}+1\right)+4\left(k \gamma_{k}-1\right)+2\left(n-k \gamma_{k}\right) \\
& =\left(n-k \gamma_{k}\right)\left(n-k \gamma_{k}+1\right)+4\left(k \gamma_{k}-1\right) .
\end{aligned}
$$

The equality holds if and only if $T-u_{i} \cong T_{n-1, k, \gamma_{k}}$ and $d_{T}(s)=\Delta=n-k \gamma_{k}$, i.e., $T \cong T_{n, k, \gamma_{k}}$.

Note that $|A|+|B|=n-1-d_{T}(s)$ and $|A| \leq \gamma_{k}$. Therefore, $|B|=n-1-d_{T}(s)-|A| \geq$ $n-1-d_{T}(s)-\gamma_{k}$ and

$$
\sum_{x s \notin E(T)} d(x) \geq|A|+2|B|=(|A|+|B|)+|B| \geq 2\left(n-1-d_{T}(s)\right)-\gamma_{k} .
$$


By the above inequality and the definition of $M_{2}$, we have

$$
\begin{aligned}
M_{2}(T)= & M_{2}\left(T-u_{i}\right)+\sum_{v \in V(T)} d_{T}(v)-\sum_{x s \notin E(T)} d_{T}(x)-1 \\
\leq & M_{2}\left(T-u_{i}\right)+2(n-1)-2\left(n-1-d_{T}(s)\right)+\gamma_{k}-1 \\
\leq & \left(n-1-k \gamma_{k}\right)\left[n-1-(k-1) \gamma_{k}\right]+(4 k-2) \gamma_{k}-4 \\
& +2\left(n-k \gamma_{k}\right)+\gamma_{k}-1 \quad\left(\text { since } d_{T}(s) \leq \Delta \leq n-k \gamma_{k}\right) \\
= & \left(n-k \gamma_{k}\right)\left[n-(k-1) \gamma_{k}\right]+(4 k-2) \gamma_{k}-4 .
\end{aligned}
$$

The equality (1) holds if and only if $|A|=\gamma_{k},|B|=n-1-d_{T}(s)-\gamma_{k}$ and $d_{T}(x)=2$ for $x \in B$. The equality (2) holds if and only if $T-u_{i} \cong T_{n-1, k, \gamma_{k}}$ and $d_{T}(s)=\Delta=n-k \gamma_{k}$. Hence, $M_{2}(T) \leq\left(n-k \gamma_{k}\right)\left[n-(k-1) \gamma_{k}\right]+(4 k-2) \gamma_{k}-4$ with equality if and only if $T \cong T_{n, k, \gamma_{k}}$.

Theorem 3.4 Let $T$ be a tree of order $n$ with distance $k$-domination number $\gamma_{k}(\geq 3)$. Then

$$
M_{1}(T) \leq\left(n-k \gamma_{k}\right)\left(n-k \gamma_{k}+1\right)+4\left(k \gamma_{k}-1\right)
$$

and

$$
M_{2}(T) \leq\left(n-k \gamma_{k}\right)\left[n-(k-1) \gamma_{k}\right]+(4 k-2) \gamma_{k}-4,
$$

with equality if and only if $T \cong T_{n, k, \gamma_{k}}$.

Proof Let $T \in \mathcal{T}_{n, k, \gamma_{k}}$ and $P=v_{0} v_{1} \cdots v_{d}$ be a diameter path of $T$. Define $S_{T}=\{u \in V(T) \mid$ $\left.d_{T}(u)=1, \gamma_{k}(T-u)=\gamma_{k}(T)\right\}$. If $S_{T}=\emptyset$, then $\gamma_{k}\left(T-v_{i}\right)=\gamma_{k}(T)-1$ for $i=0, d$. If $S_{T} \neq \emptyset$, then we suppose that $T \in\left\{T^{*} \in \mathcal{T}_{n, k, \gamma_{k}}|| N_{T^{*}}\left(S_{T^{*}}\right) \mid=1\right\}$ by Lemma 2.8 for establishing the maximum Zagreb indices of trees among $\mathcal{T}_{n, k, \gamma_{k}}$. If $v_{d} \in S_{T} \neq \emptyset$, then $\gamma_{k}\left(T-v_{0}\right)=\gamma_{k}(T)-1$, which implies that $\gamma_{k}\left(T-v_{0}\right)=\gamma_{k}(T)-1$ or $\gamma_{k}\left(T-v_{d}\right)=\gamma_{k}(T)-1$. Assume that $\gamma_{k}(T-$ $\left.v_{0}\right)=\gamma_{k}(T)-1$. Then there is a minimum distance $k$-dominating set $D$ of $T$ such that $\left\{v_{k}, v_{k+1}, v_{d-k}\right\} \subseteq D$ from the proof of Lemma 3.3.

Let $T^{\prime}$ be the tree obtained from $T$ by applying Transformation I on $T_{i}$ repeatedly for $i=$ $1, \ldots, k$ such that $T_{i}^{\prime} \cong S_{\mid V\left(T_{i}^{\prime}\right)}$, where $T_{i}^{\prime}$ is the component of $T^{\prime}-\left\{v_{i-1} v_{i}, v_{i} v_{i+1}\right\}$ containing $v_{i}, i=1, \ldots, k$ (see Figure 4). Then $T^{\prime} \in \mathcal{T}_{n, k, \gamma_{k}}$. By Lemma 2.2, we have $M_{i}(T) \leq M_{i}\left(T^{\prime}\right)$, $i=1,2$, with equality if and only if $T \cong T^{\prime}$.

By Lemma 2.3, for some $i_{0}, i_{1} \in\{1, \ldots, k\}$, define

$$
\begin{aligned}
T^{\prime \prime}= & T^{\prime}-\bigcup_{i \in\{1, \ldots, k\} \backslash\left\{i_{0}\right\}}\left\{v_{i} x \mid x \in N_{T^{\prime}}\left(v_{i}\right) \backslash\left\{v_{i-1}, v_{i+1}\right\}\right\} \\
& +\bigcup_{i \in\{1, \ldots, k\} \backslash\left\{i_{0}\right\}}\left\{v_{i_{0}} x \mid x \in N_{T^{\prime}}\left(v_{i}\right) \backslash\left\{v_{i-1}, v_{i+1}\right\}\right\}
\end{aligned}
$$




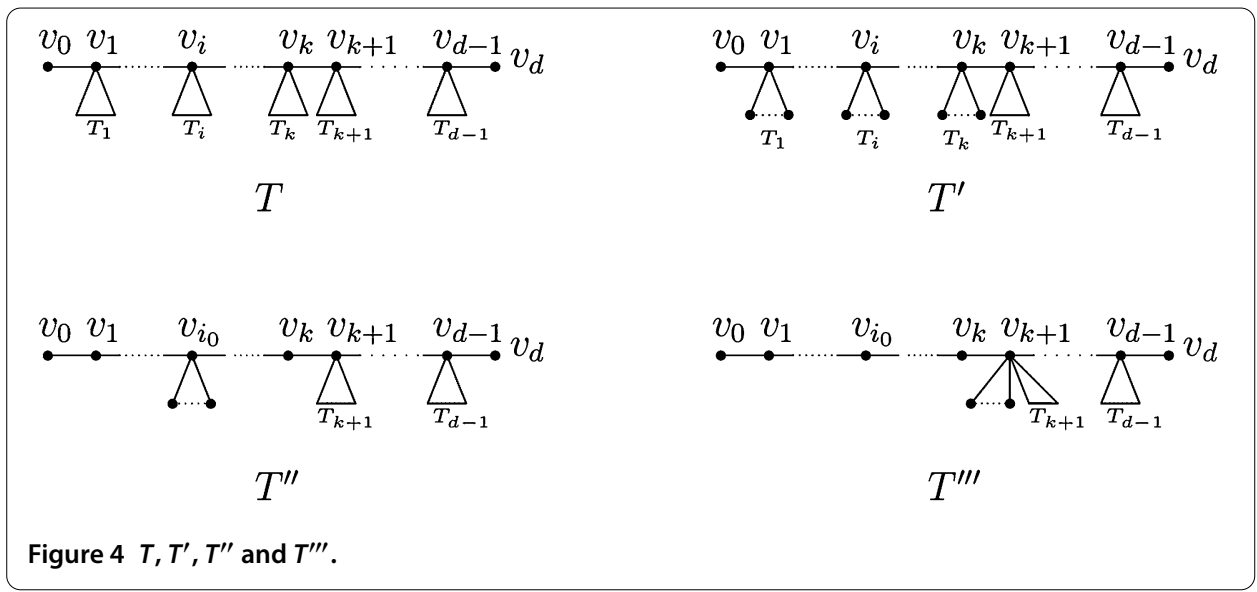

and

$$
\begin{aligned}
\widetilde{T}^{\prime \prime}= & T^{\prime}-\bigcup_{i \in\{1, \ldots, k\} \backslash\left\{i_{1}\right\}}\left\{v_{i} x \mid x \in N_{T^{\prime}}\left(v_{i}\right) \backslash\left\{v_{i-1}, v_{i+1}\right\}\right\} \\
& +\bigcup_{i \in\{1, \ldots, k\} \backslash\left\{i_{1}\right\}}\left\{v_{i_{1}} x \mid x \in N_{T^{\prime}}\left(v_{i}\right) \backslash\left\{v_{i-1}, v_{i+1}\right\}\right\} .
\end{aligned}
$$

Then one has $M_{1}\left(T^{\prime}\right) \leq M_{1}\left(T^{\prime \prime}\right)$ with equality if and only if $T^{\prime} \cong T^{\prime \prime}$ and $M_{2}\left(T^{\prime}\right) \leq M_{2}\left(\widetilde{T}^{\prime \prime}\right)$ with equality if and only if $T^{\prime} \cong \widetilde{T}^{\prime \prime}$.

Suppose that $\left|N_{T^{\prime \prime}}\left(v_{i_{0}}\right) \backslash\left\{v_{i_{0}-1}, v_{i_{0}+1}\right\}\right|=\left|N_{\widetilde{T}^{\prime \prime}} \backslash\left\{v_{i_{1}-1}, v_{i_{1}+1}\right\}\right|=m, m \geq 0$. Let

$$
\begin{aligned}
T^{\prime \prime \prime}= & T^{\prime \prime}-\left\{v_{i_{0}} x \mid x \in N_{T^{\prime \prime}}\left(v_{i_{0}}\right) \backslash\left\{v_{i_{0}-1}, v_{i_{0}+1}\right\}\right\} \\
& +\left\{v_{k+1} x \mid x \in N_{T^{\prime \prime}}\left(v_{i_{0}}\right) \backslash\left\{v_{i_{0}-1}, v_{i_{0}+1}\right\}\right\} \\
= & \widetilde{T}^{\prime \prime}-\left\{v_{i_{1}} x \mid x \in N_{\widetilde{T}^{\prime \prime}}\left(v_{i_{1}}\right) \backslash\left\{v_{i_{1}-1}, v_{i_{1}+1}\right\}\right\} \\
& +\left\{v_{k+1} x \mid x \in N_{\widetilde{T}^{\prime \prime}}\left(v_{i_{1}}\right) \backslash\left\{v_{i_{1}-1}, v_{i_{1}+1}\right\}\right\} .
\end{aligned}
$$

Then $D$ is a minimum distance $k$-dominating set of $T^{\prime \prime \prime}$ and $d_{T^{\prime \prime \prime}}\left(v_{i}\right)=2$ for $i=1, \ldots, k$. Assume that $P N_{k, D}(x)$ is the set of all private $k$-neighbors of $x$ with respect to $D$ in $T^{\prime \prime \prime}$. It is clear that the vertices in $\bigcup_{i=0}^{k} N_{T^{\prime \prime \prime}}^{i}\left(v_{k}\right) \backslash\left\{v_{0}, \ldots, v_{k}\right\}$ can be dominated by $v_{k+1} \in D$. Thus, $D \backslash\left\{v_{k}\right\}$ is a distance $k$-dominating set of tree $T^{\prime \prime \prime}-\left\{v_{0}, \ldots, v_{k}\right\}$. In addition, $P N_{k, D}\left(v_{k+1}\right) \subseteq$ $V\left(T^{\prime \prime \prime}\right) \backslash\left\{v_{0}, \ldots, v_{k}\right\}$, which means that $D \backslash\left\{v_{k}\right\}$ is a minimum distance $k$-dominating set of $T^{\prime \prime \prime}-\left\{v_{0}, \ldots, v_{k}\right\}$. So $\gamma_{k}\left(T^{\prime \prime \prime}-\left\{v_{0}, \ldots, v_{k}\right\}\right)=\gamma_{k}-1$. Analogously, $\gamma_{k}\left(T^{\prime \prime \prime}-\left\{v_{0}, \ldots, v_{k-1}\right\}\right)=$ $\gamma_{k}-1$.

By the definition of the first Zagreb index, we get

$$
\begin{aligned}
M_{1}\left(T^{\prime \prime \prime}\right)-M_{1}\left(T^{\prime \prime}\right) & =4+\left(d_{T^{\prime \prime}}\left(v_{k+1}\right)+m\right)^{2}-(2+m)^{2}-d_{T^{\prime \prime}}^{2}\left(v_{k+1}\right) \\
& =2 m\left(d_{T^{\prime \prime}}\left(v_{k+1}\right)-2\right) \\
& \geq 0
\end{aligned}
$$

so $M_{1}\left(T^{\prime \prime \prime}\right)-M_{1}\left(T^{\prime \prime}\right)=0$ if and only if at least one of the following conditions holds:

(1) $m=0$, which implies that $T^{\prime \prime} \cong T^{\prime \prime \prime}$;

(2) $d_{T^{\prime \prime}}\left(v_{k+1}\right)=2$. 
If $i_{1}=1$, then

$$
\begin{aligned}
M_{2}\left(T^{\prime \prime \prime}\right)-M_{2}\left(\widetilde{T}^{\prime \prime}\right)= & 6+\left(d_{\widetilde{T}^{\prime \prime}}\left(v_{k+1}\right)+m\right)\left(m+\sum_{x \in \widetilde{T}_{\widetilde{T}^{\prime \prime}}\left(v_{k+1}\right)} d_{\widetilde{T}^{\prime \prime}}(x)\right) \\
& -(m+2)(m+3)-d_{\widetilde{T}^{\prime \prime}}\left(v_{k+1}\right) \sum_{x \in N_{\widetilde{T}^{\prime \prime}}\left(v_{k+1}\right)} d_{\widetilde{T}^{\prime \prime}}(x) \\
= & m\left[d_{\widetilde{T}^{\prime \prime}}\left(v_{k+1}\right)+\sum_{x \in \widetilde{T}_{\widetilde{T}^{\prime \prime}}\left(v_{k+1}\right)} d_{\widetilde{T}^{\prime \prime}}(x)-5\right] \\
\geq & 0,
\end{aligned}
$$

with equality if and only if $m=0$, that is, $\widetilde{T}^{\prime \prime} \cong T^{\prime \prime \prime}$. If $i_{1} \neq 1$ and $i_{1} \neq k$, then

$$
\begin{aligned}
M_{2}\left(T^{\prime \prime \prime}\right)-M_{2}\left(\widetilde{T}^{\prime \prime}\right)= & 8+\left(d_{\widetilde{T}^{\prime \prime}}\left(v_{k+1}\right)+m\right)\left(m+\sum_{x \in N_{\widetilde{T}^{\prime \prime}}\left(v_{k+1}\right)} d_{\widetilde{T}^{\prime \prime}}(x)\right) \\
& -(m+2)(m+4)-d_{\widetilde{T}^{\prime \prime}}\left(v_{k+1}\right) \sum_{x \in N_{\widetilde{T}^{\prime \prime}}\left(v_{k+1}\right)} d_{\widetilde{T}^{\prime \prime}}(x) \\
= & m\left[d_{\widetilde{T}^{\prime \prime}}\left(v_{k+1}\right)+\sum_{x \in \widetilde{T}_{\widetilde{T}^{\prime \prime}}\left(v_{k+1}\right)} d_{\widetilde{T}^{\prime \prime}}(x)-6\right] \\
\geq & 0 .
\end{aligned}
$$

Also, $M_{2}\left(T^{\prime \prime \prime}\right)-M_{2}\left(\widetilde{T}^{\prime \prime}\right)=0$ if and only if at least one of the following conditions holds:

(1) $m=0$, namely, $\widetilde{T}^{\prime \prime} \cong T^{\prime \prime \prime}$;

(2) $d_{\widetilde{T}^{\prime \prime}}\left(v_{k}\right)=d_{\widetilde{T}^{\prime \prime}}\left(v_{k+1}\right)=d_{\widetilde{T}^{\prime \prime}}\left(v_{k+2}\right)=2$.

If $i_{1} \neq 1$ and $i_{1}=k$, then

$$
\begin{aligned}
M_{2}\left(T^{\prime \prime \prime}\right)-M_{2}\left(\widetilde{T}^{\prime \prime}\right)= & 4+\left(d_{\widetilde{T}^{\prime \prime}}\left(v_{k+1}\right)+m\right)\left(m+2+\sum_{x \in N_{\widetilde{T}^{\prime \prime}}\left(v_{k+1}\right) \backslash\left\{v_{k}\right\}} d_{\widetilde{T}^{\prime \prime}}(x)\right) \\
& -(m+2)(m+2)-d_{\widetilde{T}^{\prime \prime}}\left(v_{k+1}\right)\left(\sum_{x \in N_{\widetilde{T}^{\prime \prime}}\left(v_{k+1}\right) \backslash\left\{v_{k}\right\}} d_{\widetilde{T}^{\prime \prime}}(x)+m+2\right) \\
= & m\left(\sum_{x \in N_{\widetilde{T}}^{\prime \prime}\left(v_{k+1}\right) \backslash\left\{v_{k}\right\}} d_{\widetilde{T}^{\prime \prime}}(x)-2\right) \\
\geq & 0 .
\end{aligned}
$$

As a result, $M_{2}\left(T^{\prime \prime \prime}\right)-M_{2}\left(\widetilde{T}^{\prime \prime}\right)=0$ if and only if at least one of the following conditions holds:

(1) $m=0$, which implies that $\widetilde{T}^{\prime \prime} \cong T^{\prime \prime \prime}$;

(2) $d_{\widetilde{T}^{\prime \prime}}\left(v_{k+1}\right)=d_{\widetilde{T}^{\prime \prime}}\left(v_{k+2}\right)=2$.

In what follows, we prove $M_{1}\left(T^{\prime \prime \prime}\right) \leq\left(n-k \gamma_{k}\right)\left(n-k \gamma_{k}+1\right)+4\left(k \gamma_{k}-1\right)$ and $M_{2}\left(T^{\prime \prime \prime}\right) \leq$ $\left(n-k \gamma_{k}\right)\left[n-(k-1) \gamma_{k}\right]+(4 k-2) \gamma_{k}-4$ with equality if and only if $T^{\prime \prime \prime} \cong T_{n, k, \gamma_{k}}$ by induction on $\gamma_{k}$. The statement is true for $\gamma_{k}=3$ and $n \geq(k+1) \gamma_{k}$ by Lemma 3.3. Assume that $\gamma_{k} \geq 4$, the statement holds for $\gamma_{k}-1$ and all the $n \geq(k+1)\left(\gamma_{k}-1\right)$. 
In view of $\gamma_{k}\left(T^{\prime \prime \prime}-\left\{v_{0}, v_{1}, \ldots, v_{k}\right\}\right)=\gamma_{k}-1$ and $\left|V\left(T^{\prime \prime \prime}-\left\{v_{0}, v_{1}, \ldots, v_{k}\right\}\right)\right|=n-k-1 \geq$ $(k+1)\left(\gamma_{k}-1\right)$, by the induction hypothesis, we get

$$
\begin{aligned}
M_{1}\left(T^{\prime \prime \prime}\right) & =M_{1}\left(T^{\prime \prime \prime}-\left\{v_{0}, v_{1}, \ldots, v_{k}\right\}\right)+2 d_{T^{\prime \prime \prime}}\left(v_{k+1}\right)-1+\sum_{i=0}^{k} d_{T^{\prime \prime \prime}}^{2}\left(v_{i}\right) \\
& \leq M_{1}\left(T_{n-k-1, k, \gamma_{k}-1}\right)+2\left(n-k \gamma_{k}\right)+4 k \\
& =\left(n-k \gamma_{k}\right)\left(n-k \gamma_{k}+1\right)+4\left(k \gamma_{k}-1\right) .
\end{aligned}
$$

The equality holds if and only if $T^{\prime \prime \prime}-\left\{v_{0}, v_{1}, \ldots, v_{k}\right\} \cong T_{n-k-1, k, \gamma_{k}-1}$ and $d_{T^{\prime \prime \prime}}\left(v_{k+1}\right)=\Delta=$ $n-k \gamma_{k}$. Recalling that $d_{T^{\prime \prime \prime}}\left(v_{i}\right)=2$ for $i=1, \ldots, k$, we have $M_{1}\left(T^{\prime \prime \prime}\right)=\left(n-k \gamma_{k}\right)\left(n-k \gamma_{k}+1\right)+$ $4\left(k \gamma_{k}-1\right)$ if and only if $T^{\prime \prime \prime} \cong T_{n, k, \gamma_{k}}$.

Thus, $M_{1}(T) \leq M_{1}\left(T^{\prime}\right) \leq M_{1}\left(T^{\prime \prime}\right) \leq M_{1}\left(T^{\prime \prime \prime}\right) \leq\left(n-k \gamma_{k}\right)\left(n-k \gamma_{k}+1\right)+4\left(k \gamma_{k}-1\right)$ and $M_{1}(T)=\left(n-k \gamma_{k}\right)\left(n-k \gamma_{k}+1\right)+4\left(k \gamma_{k}-1\right)$ if and only if at least one of the following conditions holds:

(1) $T \cong T^{\prime} \cong T^{\prime \prime} \cong T^{\prime \prime \prime} \cong T_{n, k, \gamma_{k}}$;

(2) $T \cong T^{\prime} \cong T^{\prime \prime}$, where $d_{T^{\prime \prime}}\left(v_{k+1}\right)=2$. Besides, $T^{\prime \prime \prime} \cong T_{n, k, \gamma_{k}}$.

However, the second condition is impossible. If $T^{\prime \prime \prime} \cong T_{n, k, \gamma_{k}}$, then $d_{T^{\prime \prime \prime}}\left(v_{k+1}\right)=n-k \gamma_{k}$ and the number of the pendent vertices in $N_{T^{\prime \prime \prime}}\left(v_{k+1}\right)$ is $n-(k+1) \gamma_{k}$. By the definition of $T^{\prime \prime \prime}$, we have

$$
n-(k+1) \gamma_{k} \geq\left|N_{T^{\prime \prime}}\left(v_{i_{0}}\right) \backslash\left\{v_{i_{0}-1}, v_{i_{0}+1}\right\}\right|
$$

Hence,

$$
\begin{aligned}
d_{T^{\prime \prime}}\left(v_{k+1}\right) & =d_{T^{\prime \prime \prime}}\left(v_{k+1}\right)-\left|N_{T^{\prime \prime}}\left(v_{i_{0}}\right) \backslash\left\{v_{i_{0}-1}, v_{i_{0}+1}\right\}\right| \\
& \geq d_{T^{\prime \prime \prime}}\left(v_{k+1}\right)-\left[n-(k+1) \gamma_{k}\right] \\
& =\gamma_{k} \geq 3
\end{aligned}
$$

a contradiction to $d_{T^{\prime \prime}}\left(v_{k+1}\right)=2$. Therefore,

$$
M_{1}(T) \leq\left(n-k \gamma_{k}\right)\left(n-k \gamma_{k}+1\right)+4\left(k \gamma_{k}-1\right)
$$

with equality if and only if $T \cong T_{n, k, \gamma_{k}}$.

Note that $\gamma_{k}\left(T^{\prime \prime \prime}-\left\{v_{0}, \ldots, v_{k-1}\right\}\right)=\gamma_{k}-1$ and $\left|V\left(T^{\prime \prime \prime}-\left\{v_{0}, \ldots, v_{k-1}\right\}\right)\right|>(k+1)\left(\gamma_{k}-1\right)$. Then

$$
\begin{aligned}
M_{2}\left(T^{\prime \prime \prime}\right) & =M_{2}\left(T^{\prime \prime \prime}-\left\{v_{0}, v_{1}, \ldots, v_{k-1}\right\}\right)+d_{T^{\prime \prime \prime}}\left(v_{k+1}\right)+4(k-1)+2 \\
& \leq M_{2}\left(T_{n-k, k, \gamma_{k}-1}\right)+n-k \gamma_{k}+4(k-1)+2 \\
& =\left(n-k \gamma_{k}\right)\left[n-(k-1) \gamma_{k}\right]+(4 k-2) \gamma_{k}-4 .
\end{aligned}
$$

The equality holds if and only if $T^{\prime \prime \prime}-\left\{v_{0}, \ldots, v_{k-1}\right\} \cong T_{n-k, k, \gamma_{k}-1}$ and $d_{T^{\prime \prime \prime}}\left(v_{k+1}\right)=\Delta=n-k \gamma_{k}$. In consideration of $d_{T^{\prime \prime \prime}}\left(v_{i}\right)=2$ for $i=1, \ldots, k$, the equality holds if and only if $T^{\prime \prime \prime} \cong T_{n, k, \gamma_{k}}$. Hence, if $i_{1} \neq 1$, then $M_{2}(T) \leq M_{2}\left(T^{\prime}\right) \leq M_{2}\left(\widetilde{T}^{\prime \prime}\right) \leq M_{2}\left(T^{\prime \prime \prime}\right) \leq\left(n-k \gamma_{k}\right)\left[n-(k-1) \gamma_{k}\right]+$ $(4 k-2) \gamma_{k}-4$, with equality if and only if at least one of the following conditions holds: 
(1) $T \cong T^{\prime} \cong \widetilde{T}^{\prime \prime} \cong T^{\prime \prime \prime} \cong T_{n, k, \gamma_{k}}$;

(2) $T \cong T^{\prime} \cong \widetilde{T}^{\prime \prime}$, where $d_{\widetilde{T}^{\prime \prime}}\left(v_{k}\right)=d_{\widetilde{T}^{\prime \prime}}\left(v_{k+1}\right)=d_{\widetilde{T}^{\prime \prime}}\left(v_{k+2}\right)=2$ and $\widetilde{T}^{\prime \prime \prime} \cong T_{n, k, \gamma_{k}}$.

Analogous to the analysis of the first Zagreb index, the second condition above is impossible. Thus,

$$
M_{2}(T) \leq\left(n-k \gamma_{k}\right)\left[n-(k-1) \gamma_{k}\right]+(4 k-2) \gamma_{k}-4
$$

and the equality holds if and only if $T \cong T_{n, k, \gamma_{k}}$.

Besides, if $i=1$, then $M_{2}(T) \leq\left(n-k \gamma_{k}\right)\left[n-(k-1) \gamma_{k}\right]+(4 k-2) \gamma_{k}-4$ with equality if and only if $T \cong T_{n, k, \gamma_{k}}$ immediately. This completes the proof.

Remark 3.5 Borovićanin and Furtula [1] proved

$$
M_{1}(T) \leq(n-\gamma)(n-\gamma+1)+4(\gamma-1)
$$

and

$$
M_{2}(T) \leq 2(n-\gamma+1)(\gamma-1)+(n-\gamma)(n-2 \gamma+1)
$$

with equality if and only if $T \cong T_{n, \gamma}$, where $T_{n, \gamma}$ is the tree obtained from the $\operatorname{star} K_{1, n-\gamma}$ by attaching a pendent edge to its $\gamma-1$ pendent vertices. In this paper, we determine the extremal values on the Zagreb indices of trees with distance $k$-domination number for $k \geq 2$. Note that the domination number is the special case of the distance $k$-domination number for $k=1$ and $T_{n, k, \gamma_{k}} \cong T_{n, \gamma}, T_{n, k, 2}^{i} \cong T_{n, \gamma}, i \in\{1, \ldots, k\}$, when $k=1$. Let $T$ be an $n$-vertex tree with distance $k$-domination number $\gamma_{k}$. Then, by using Theorems 3.1, 3.2 and 3.4 and the results in [1], we have

$$
M_{1}(T) \leq \begin{cases}n(n-1) & \text { if } \gamma_{k}=1 \\ \left(n-k \gamma_{k}\right)\left(n-k \gamma_{k}+1\right)+4\left(k \gamma_{k}-1\right) & \text { if } \gamma_{k} \geq 2\end{cases}
$$

with equality if and only if $T \cong S_{n}$ when $\gamma_{k}=1, T \cong T_{n, k, 2}^{i}, i \in\{1, \ldots, k\}$, when $\gamma_{k}=2$, or $T \cong T_{n, k, \gamma_{k}}$ when $\gamma_{k} \geq 3$. Moreover,

$$
M_{2}(T) \leq \begin{cases}2\left(n-\gamma_{k}+1\right)\left(\gamma_{k}-1\right)+\left(n-\gamma_{k}\right)\left(n-2 \gamma_{k}+1\right) & \text { if } k=1, \\ (n-1)^{2} & \text { if } k \geq 2, \gamma_{k}=1, \\ \left(n-k \gamma_{k}\right)\left[n-(k-1) \gamma_{k}\right]+(4 k-2) \gamma_{k}-4 & \text { if } k \geq 2, \gamma_{k} \geq 2,\end{cases}
$$

with equality if and only if $T \cong S_{n}$ when $k \geq 2$ and $\gamma_{k}=1, T \cong T_{n, k, 2}^{i}, i \in\{2, \ldots, k\}$, when $k \geq 2$ and $\gamma_{k}=2$, or $T \cong T_{n, k, \gamma_{k}}$ otherwise.

\section{Appendix}

Proof Either $M_{i}\left(G_{1}\right)>M_{i}(G)$ or $M_{i}\left(G_{2}\right)>M_{i}(G), i=1,2$, in Lemma 2.8, where $G_{1}=G$ $\left\{v y_{1}\right\}+\left\{u y_{1}\right\}$ and $G_{2}=G-\left\{u x_{1}\right\}+\left\{v x_{1}\right\}$, as shown in the following figure. 


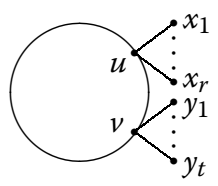

G

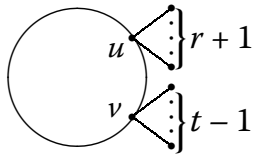

$G_{1}$

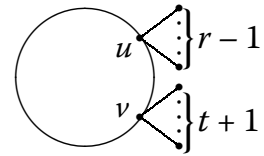

$G_{2}$

Let $G^{*}=G-\left\{x_{1}, \ldots, x_{r}, y_{1}, \ldots, y_{t}\right\}, d_{G^{*}}(u)=a$ and $d_{G^{*}}(v)=b$. Then

$$
\begin{aligned}
M_{1}\left(G_{1}\right)-M_{1}(G) & =(a+r+1)^{2}+(b+t-1)^{2}-(a+r)^{2}-(b+t)^{2} \\
& =2(a+r-b-t+1)
\end{aligned}
$$

and

$$
\begin{aligned}
M_{1}\left(G_{2}\right)-M_{1}(G) & =(a+r-1)^{2}+(b+t+1)^{2}-(a+r)^{2}-(b+t)^{2} \\
& =2(b+t-a-r+1)
\end{aligned}
$$

by the definition of the first Zagreb index. Suppose that $M_{1}\left(G_{1}\right)-M_{1}(G) \leq 0$. Then $a+r \leq$ $b+t-1$. It follows that $M_{1}\left(G_{2}\right)-M_{1}(G)>0$.

If $u \notin N_{G}(v)$, then

$$
\begin{aligned}
M_{2}\left(G_{1}\right)-M_{2}(G)= & (a+r+1)\left(\sum_{x \in N_{G^{*}}(u)} d_{G}(x)+r+1\right) \\
& +(b+t-1)\left(\sum_{x \in N_{G^{*}}(v)} d_{G}(x)+t-1\right) \\
& -(a+r)\left(\sum_{x \in N_{G^{*}}(u)} d_{G}(x)+r\right)-(b+t)\left(\sum_{x \in N_{G^{*}}(v)} d_{G}(x)+t\right) \\
= & \sum_{x \in N_{G^{*}(u)}} d_{G}(x)-\sum_{x \in N_{G^{*}}(v)} d_{G}(x)+2 r-2 t+a-b+2
\end{aligned}
$$

and

$$
\begin{aligned}
M_{2}\left(G_{2}\right)-M_{2}(G)= & (a+r-1)\left(\sum_{x \in N_{G^{*}}(u)} d_{G}(x)+r-1\right) \\
& +(b+t+1)\left(\sum_{x \in N_{G^{*}}(v)} d_{G}(x)+t+1\right) \\
& -(a+r)\left(\sum_{x \in N_{G^{*}}(u)} d_{G}(x)+r\right)-(b+t)\left(\sum_{x \in N_{G^{*}}(v)} d_{G}(x)+t\right) \\
= & \sum_{x \in N_{G^{*}}(v)} d_{G}(x)-\sum_{x \in N_{G^{*}}(u)} d_{G}(x)+2 t-2 r+b-a+2 .
\end{aligned}
$$

If $M_{2}\left(G_{1}\right)-M_{2}(G) \leq 0$, then $M_{2}\left(G_{2}\right)-M_{2}(G)>0$. 
If $u \in N_{G}(v)$, then

$$
\begin{aligned}
& M_{2}\left(G_{1}\right)-M_{2}(G) \\
& =(a+r+1)\left(\sum_{x \in N_{G^{*}}(u) \backslash\{v\}} d_{G}(x)+r+1\right)+(b+t-1)\left(\sum_{x \in N_{G^{*}}(u) \backslash\{v\}} d_{G}(x)+t-1\right) \\
& \quad+(a+r+1)(b+t-1)-(a+r)\left(\sum_{x \in N_{G^{*}}(u) \backslash\{v\}} d_{G}(x)+r\right) \\
& \quad-(b+t)\left(\sum_{x \in N_{G^{*}}(u) \backslash\{v\}} d_{G}(x)+t\right)-(a+r)(b+t) \\
& =\sum_{x \in N_{G^{*}}(u) \backslash\{v\}} d_{G}(x)-\sum_{x \in N_{G^{*}}(v) \backslash\{u\}} d_{G}(x)+r-t+1
\end{aligned}
$$

and

$$
\begin{aligned}
M_{2}\left(G_{2}\right)-M_{2}(G) & =(a+r-1)\left(\sum_{x \in N_{G^{*}}(u) \backslash\{v\}} d_{G}(x)+r-1\right)+(b+t+1)\left(\sum_{x \in N_{G^{*}}(u) \backslash\{v\}} d_{G}(x)+t+1\right) \\
& +(a+r-1)(b+t+1)-(a+r)\left(\sum_{x \in N_{G^{*}}(u) \backslash\{v\}} d_{G}(x)+r\right) \\
& -(b+t)\left(\sum_{x \in N_{G^{*}}(u) \backslash\{v\}} d_{G}(x)+t\right)-(a+r)(b+t) \\
= & \sum_{x \in N_{G^{*}}(v) \backslash\{u\}} d_{G}(x)-\sum_{x \in N_{G^{*}}(u) \backslash\{v\}} d_{G}(x)+t-r+1 .
\end{aligned}
$$

Assume that $M_{2}\left(G_{1}\right)-M_{2}(G) \leq 0$. Then $M_{2}\left(G_{2}\right)-M_{2}(G)>0$. Therefore, either $M_{i}\left(G_{1}\right)>$ $M_{i}(G)$ or $M_{i}\left(G_{2}\right)>M_{i}(G), i=1,2$.

\section{Acknowledgements}

This work is financially supported by the National Natural Science Foundation of China (No. 11401004) and the Natural Science Foundation of Anhui Province of China (No. 1408085QA03).

\section{Competing interests}

The authors declare that they have no competing interests.

\section{Authors' contributions}

All authors read and approved the final manuscript.

\section{Publisher's Note}

Springer Nature remains neutral with regard to jurisdictional claims in published maps and institutional affiliations.

Received: 25 September 2017 Accepted: 15 December 2017 Published online: 10 January 2018

\section{References}

1. Borovićanin, B, Furtula, B: On extremal Zagreb indices of trees with given domination number. Appl. Math. Comput. 279, 208-218 (2016)

2. Dobrynin, A, Kochetova, AA: Degree distance of a graph: a degree analogue of the Wiener index. J. Chem. Inf. Comput. Sci. 34, 1082-1086 (1994)

3. Dobrynin, A, Entringer, R, Gutman, I: Wiener index of trees: theory and applications. Acta Appl. Math. 66, 211-249 (2011) 
4. Balaban, AT, Chiriac, A, Motoc, I, Simon, Z: Steric Fit in Quantitative Structure-Activity Relations. Lecture Notes in Chemistry, vol. 15, pp. 22-27. Springer, Berlin (1980)

5. Estrada, E: Characterization of 3D molecular structure. Chem. Phys. Lett. 319, 713-718 (2000)

6. Zhang, HP, Yang, YJ: Resistance distance and Kirchhoff index in circulant graphs. Int. J. Quant. Chem. 107, 330-339 (2007)

7. Bordes, A, Glorot, X, Weston, J, Bengio, Y: A semantic matching energy function for learning with multi-relational data Mach. Learn. 94, 233-259 (2014)

8. Gupta, S, Singh, M, Madan, AK: Eccentric distance sum: a novel graph invariant for predicting biological and physical properties. J. Math. Anal. Appl. 275, 386-401 (2002)

9. Gupta, S, Singh, M, Madan, AK: Connective eccentricity index: a novel topological descriptor for predicting biological activity. J. Mol. Graph. Model. 18, 18-25 (2000)

10. Sardana, S, Madan, AK: Predicting anti-HIV activity of TIBO derivatives: a computational approach using a novel topological descriptor. J. Mol. Model. 8, 258-265 (2002)

11. Randić, M: On characterization of molecular branching. J. Am. Chem. Soc. 97, 6609-6615 (1975)

12. Shi, YT: Note on two generalizations of the Randic index. Appl. Math. Comput. 265, 1019-1025 (2015)

13. Lokesha, V, Shetty, BS, Ranjini, PS, Cangul, IN, Cevilk, AS: New bounds for Randic and GA indices. J. Inequal. Appl. 2013, Article ID 180 (2013)

14. Zhou, B, Trinajstić, N: On general sum-connectivity index. J. Math. Chem. 47, 210-218 (2013)

15. Akhter, S, Imran, M, Raza, Z: Bounds for the general sum-connectivity index of composite graphs. J. Inequal. Appl. 2017, Article ID 76 (2017)

16. Gutman, I, Trinajstić, N: Graph theory and molecular orbitals. Total $\pi$-electron energy of alternant hydrocarbons. Chem. Phys. Lett. 17, 535-538 (1972)

17. Gutman, I: Multiplicative Zagreb indices of trees. Bull. Soc. Math. Banja Luka 18, 17-23 (2011)

18. Das, KC, Yurttas, A, Togan, M, Cevik, AS, Cangul, IN: The multiplicative Zagreb indices of graph operations. J. Inequal. Appl. 2013, Article ID 90 (2013)

19. Zhan, FQ, Qiao, YF, Cai, JL: Unicyclic and bicyclic graphs with minimal augmented Zagreb index. J. Inequal. Appl. 2015, Article ID 126 (2015)

20. Balaban, AT, Motoc, I, Bonchev, D, Mekenyan, O: Topological indices for structure-activity correlations. Top. Curr. Chem. 114, 21-55 (1983)

21. Todeschini, R, Consonni, V: Handbook of Molecular Descriptors. Wiley-VCH, Weinheim (2000)

22. Gutman, I, Ruščić, B, Trinajstić, N, Wilcox, CF: Graph theory and molecular orbitals, XII. Acyclic polyencs. J. Chem. Phys. 62, 3399-3405 (1975)

23. Nikolić, S, Kovaćević, G, Miličević, A, Trinajstić, N: The Zagreb indices 30 years after. Croat. Chem. Acta 76, 113-124 (2003)

24. Gutman, I, Das, KC: The first Zagreb index 30 years after. MATCH Commun. Math. Comput. Chem. 50, 83-92 (2004)

25. Furtula, B, Gutman, I: A forgotten topological index. J. Math. Chem. 53, 1184-1190 (2015)

26. Hosamani, SM, Basavanagoud, B: New upper bounds for the first Zagreb index. MATCH Commun. Math. Comput. Chem. 74, 97-101 (2015)

27. Milovanović, El, Milovanović, IŽ, Dolićanin, EĆ, Glogić, E: A note on the first reformulated Zagreb index. Appl. Math. Comput. 273, 16-20 (2016)

28. Furtula, B, Gutmana, I, Ediz, S: On difference of Zagreb indices. Discrete Appl. Math. 178, 83-88 (2014)

29. Liu, BL, Gutman, I: Upper bounds for Zagreb indices of connected graphs. MATCH Commun. Math. Comput. Chem. $55,439-446(2006)$

30. Zhang, SG, Zhang, HL: Unicyclic graphs with the first three smallest and largest first general Zagreb index. MATCH Commun. Math. Comput. Chem. 55, 427-438 (2006)

31. Zhou, B, Gutman, I: Further properties of Zagreb indices. MATCH Commun. Math. Comput. Chem. 54, $233-239$ (2005)

32. Gutman, I, Goubko, M: Trees with fixed number of pendent vertices with minimal first Zagreb index. Bull. Int. Math. Virtual Inst. 3, 161-164 (2013)

33. Yan, Z, Liu, HQ, Liu, HG: Sharp bounds for the second Zagreb index of unicyclic graphs. J. Math. Chem. 42, 565-574 (2007)

34. Lang, RL, Deng, XL, Lu, H: Bipartite graphs with the maximal value of the second Zagreb index. Bull. Malays. Math. Sci. Soc. 36, 1-6 (2013)

35. Vasilyeva, A, Dardab, R, Stevanović, D: Trees of given order and independence number with minimal first Zagreb index. MATCH Commun. Math. Comput. Chem. 72, 775-782 (2014)

36. Feng, YQ, Hu, X, Li, SC: On the extremal Zagreb indices of graphs with cut edges. Acta Appl. Math. 10, 667-684 (2010)

37. Liu, MH, Liu, BL: Second Zagreb indices of unicyclic graphs with given degree sequences. Discrete Appl. Math. 167, 217-221 (2014)

38. Xu, KX: The Zagreb indices of graphs with a given clique number. Appl. Math. Lett. 24, 1026-1030 (2011)

39. Li, SC, Yang, HX, Zhao, Q: Sharp bounds on Zagreb indices of cacti with $k$ pendent vertices. Filomat 26, 1189-1200 (2012)

40. Li, SC, Zhang, MJ: Sharp bounds for the Zagreb indices of bipartite graphs with a given diameter. Appl. Math. Lett. 24 131-137 (2011)

41. Chang, GJ: k-domination and graph covering problems. Ph.D. thesis, School of OR and IE, Cornell University, Ithaca, NY (1982)

42. Chang, GJ, Nemhauser, GL: The k-domination and k-stability problems on sun-free chordal graphs. SIAM J. Algebraic Discrete Methods 5, 332-345 (1984)

43. Haynes, TW, Hedetniemi, ST, Slater, PJ: Fundamentals of Domination in Graphs. Marcel Dekker, New York (1998)

44. Haynes, TW, Hedetniemi, ST, Slater, PJ: Domination in Graphs. Marcel Dekker, New York (1998)

45. Dankelmann, P: Average distance and domination number. Discrete Appl. Math. 80, 21-35 (1997)

46. Tian, F, Xu, JM: Average distance and distance domination numbers. Discrete Appl. Math. 157, 1113-1127 (2009)

47. $\mathrm{He}, \mathrm{CX}, \mathrm{Wu}, \mathrm{BF}, \mathrm{Yu}, \mathrm{ZS}$ : On the energy of trees with given domination number. MATCH Commun. Math. Comput. Chem. 64, 169-180 (2010) 
48. Das, KC, Gutman, I: Some properties of the second Zagreb index. MATCH Commun. Math. Comput. Chem. 52, 103-112 (2004)

49. Hua, HB, Zhang, SG, Xu, KX: Further results on the eccentric distance sum. Discrete Appl. Math. 160, 170-180 (2012)

50. Deng, HY: A unified approach to the extremal Zagreb indices for trees, unicyclic graphs and bicyclic graphs. MATCH Commun. Math. Comput. Chem. 57, 597-616 (2007)

51. Meir, A, Moon, JW: Relations between packing and covering numbers of a tree. Pac. J. Math. 61, 225-233 (1975)

52. Topp, J, Volkmann, L: On packing and covering numbers of graphs. Discrete Math. 96, 229-238 (1991)

Submit your manuscript to a SpringerOpen ${ }^{\circ}$ journal and benefit from:

- Convenient online submission

- Rigorous peer review

- Open access: articles freely available online

- High visibility within the field

- Retaining the copyright to your article

Submit your next manuscript at $\gg$ springeropen.com 